\title{
Nicorandil was an Effective Treatment Option for a Patient with Bland-White-Garland Syndrome
}

\author{
Shun Morishita, Hirofumi Maeba, Kazuya Takehana and Ichiro Shiojima
}

\begin{abstract}
:
A 75-year-old woman who had previously been diagnosed with Bland-White-Garland syndrome was admitted to our hospital for acute decompensated heart failure (ADHF). Following her recovery from ADHF, pharmacologic stress myocardial scintigraphy revealed myocardial ischemia in the basal anterior area of the left ventricle. Moreover, myocardial scintigraphy showed the improvement of the myocardial ischemia after 6 months of nicorandil administration.
\end{abstract}

Key words: Bland-White-Garland syndrome, coronary steal, nicorandil

(Intern Med 56: 2295-2299, 2017)

(DOI: 10.2169/internalmedicine.8516-16)

\section{Introduction}

A left coronary artery originating from the pulmonary trunk, also known as Bland-White-Garland (BWG) syndrome, is a rare congenital anomaly that accounts for $0.5 \%$ of cases of congenital heart disease (1). Lethal myocardial ischemia is not rare and some patients remain asymptomatic until adulthood (2). We herein present a case of acute decompensated heart failure (ADHF) with myocardial ischemia in an adult patient with BWG. We also assessed the efficacy of nicorandil in the treatment of myocardial ischemia using scintigraphy.

\section{Case Report}

In July 2013, a 75-year-old woman with dyspnea was admitted to our department. She did not complain of obvious chest pain or chest oppression. At 55 years of age she was diagnosed with BWG syndrome at another hospital. She had a medical history of dyslipidemia and chronic atrial fibrillation. Her consciousness level on admission was clear. A physical examination showed a distended neck vein and a bilateral leg edema with unremarkable heart and lung sounds. She had an irregular heart rate of 135 beats $/ \mathrm{min}$, and her arterial blood pressure was $105 / 72 \mathrm{mmHg}$. A 12lead electrocardiogram showed atrial fibrillation with a rapid ventricular response, and ST depression in the I aVL V3-V 6. Moreover, a chest X-ray revealed cardiomegaly with pulmonary congestion and pleural effusion (Fig. 1).

Transthoracic echocardiography using the modified Simpson's method demonstrated a normal systolic function with an ejection fraction of $63 \%$. Newly developed mild hypokinesis, which had not been detected when we previously performed transthoracic echocardiography, was shown in the left ventricular (LV) basal-mid anterior area.

The visualized dilated left anterior descending coronary artery in the septal area had the same appearance on the four-chamber view as it did on transthoracic echocardiography, which had been performed prior to admission. While the common features of adult BWG syndrome, such as papillary dysfunction and left ventricular aneurysm were not documented, mild-to-moderate mitral regurgitation was observed due to the reduced mobilization of the posterior mitral leaflet.

We continued to prescribe enarapril (2.5 mg/day), trasemide ( $4 \mathrm{mg} /$ day), and an oral vitamin $\mathrm{K}$ antagonist, as per the patient's pre-admission prescription. Moreover, we prescribed the intravenous administration of a vasodilator and diuretics based on the diagnosis of ADHF. The patient's symptoms improved after a few days.

Angiography performed after the patient's recovery from ADHF showed that the anomalous origin of the left anterior descending artery, which originated from the pulmonary ar- 


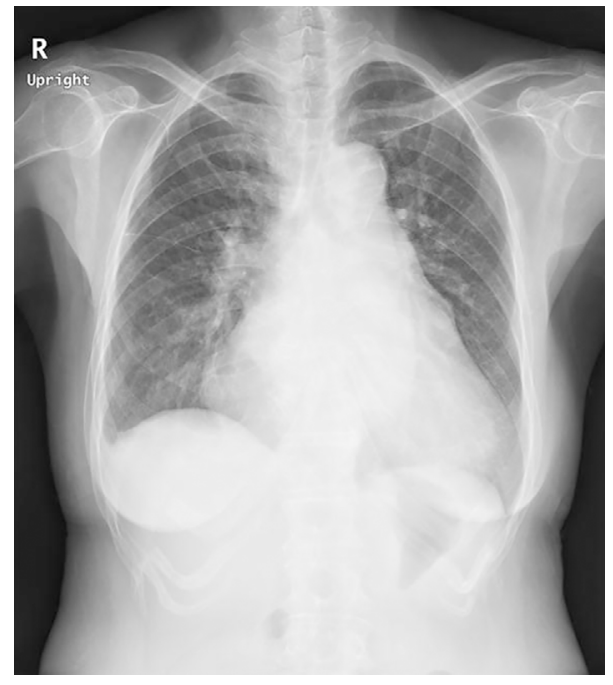

Figure 1. Chest X-ray revealing cardiomegaly with pulmonary congestion and pleural effusion.

tery, and an enlarged right coronary artery, which arose normally from the aorta, with retrograde filling of the left coronary artery and complicated collateral circulation (Fig. 2). Thus, the patient was diagnosed with typical BWG syndrome. Angiography showed no difference from coronary angiography that had been performed 6 years previously.

As the cause of the newly developed anterior mild hypokinesis could not be determined from angiography, we performed myocardial perfusion using adenosine stress and rest imaging with a ${ }^{99 \mathrm{~m}} \mathrm{Tc}$-tetrofosmin single-photon emission computed tomography (SPECT). The adenosine stress-rest ${ }^{99 \mathrm{~m}}$ Tc-tetrofosmin SPECT study revealed moderately reduced perfusion in the LV anterior area on a stress image and mildly reduced perfusion on the rest image. This incomplete recovery of perfusion in the LV anterior area suggested silent anterior myocardial ischemia and myocardial damage that matched the area of the wall motion abnormality (Fig. 3). These findings implied longstanding demand ischemia and a consequent reduction of the viable myocardium in the LV anterior area without significant organic stenosis, which had been observed on coronary angiography. Although it was recommended, the patient refused to undergo coronary bypass graft surgery.

After her ADHF improved, we added bisoprolol (1.25 $\mathrm{mg} /$ day) to the patient's regimen. However, we could not continue to prescribe beta blockers, as her heart rate had fallen to less than 60 beats per minute with symptoms such as dizziness and fatigue. We therefore added nicorandil (15 $\mathrm{mg}$ /day) to reduce the demand ischemia. After 6 months of treatment, we performed adenosine stress-rest ${ }^{99 \mathrm{~m}} \mathrm{Tc}$ tetrofosmin SPECT to evaluate the efficacy of the nicorandil. Adenosine stress-rest ${ }^{99 \mathrm{~m}} \mathrm{Tc}$-tetrofosmin SPECT performed after 6 months of treatment revealed a mild fixed defect in the LV anterior area (Fig. 4). The stress-induced ischemia in the anterior area showed an improvement in comparison to the stress-induced ischemia observed on the image taken 6 months previously, indicating that nicorandil

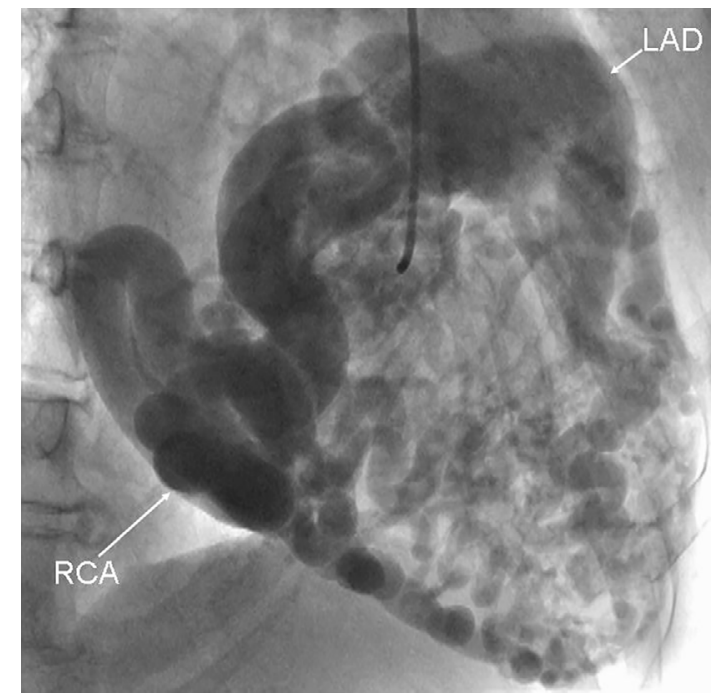

Figure 2. Angiography showing the enlarged right coronary artery arising normally from the aorta, with retrograde filling of the left coronary artery and complicated collateral circulation. RCA: right coronary artery, LAD: left anterior descending coronary artery

can act as an anti-ischemic drug in patients with BWG syndrome. On the other hand, transthoracic echocardiography did not show the normalization of the basal mid-anterior wall of the left ventricle, which had shown hypokinetic motion 6 months previously.

The N-terminal pro-brain natriuretic peptide (NT proBNP) value at discharge was $2,686 \mathrm{pg} / \mathrm{mL}$. In contrast, the value at 6 months after discharge was $2,272 \mathrm{pg} / \mathrm{mL}$.

\section{Discussion}

In the present case, we believe that nicorandil worked as an anti-ischemic agent in BWG syndrome; however, the improvement of myocardial perfusion in the area with ischemia due to BWG syndrome was modest. In patients with BWG syndrome, myocardial ischemia is caused by an increase in the size of the arteriovenous fistula and the stealing of blood from the left coronary distribution without organic stenosis of the epicardial coronary artery (3).

We hypothesize that adenosine pharmacologic stressinduced myocardial ischemia occurs due to the deterioration of the coronary steal phenomenon caused by hyperemia in patients with BWG syndrome. On the other hand, the action of nicorandil is divided into two major roles: a vasoactive effect and a direct effect on the intercellular mitochondria via the actions of nitrates and ATP-sensitive potassium channel (K-ATP channel) openers.

It is therefore necessary to discuss why the administration of nicorandil can protect the myocardium from ischemic damage during pharmacologic stress testing with adenosine. First, the rapid vasoactive effect of nicorandil should be discussed. Nicorandil can dilate the large coronary artery via the vasodilatory effect of nitrates and small resistant coro- 


\section{Before nicorandil administration}

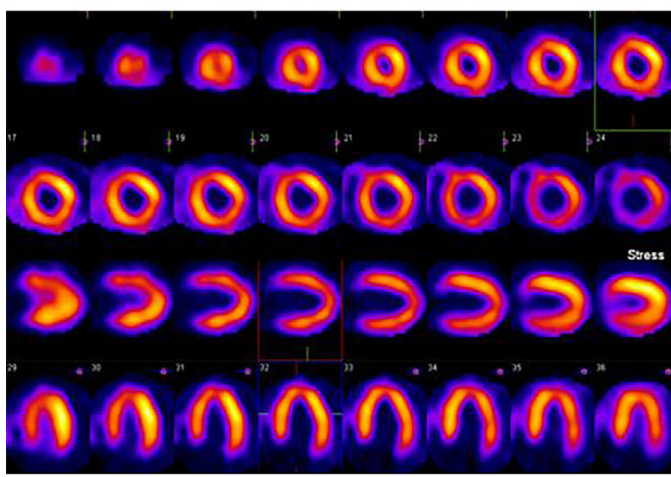

Stress image

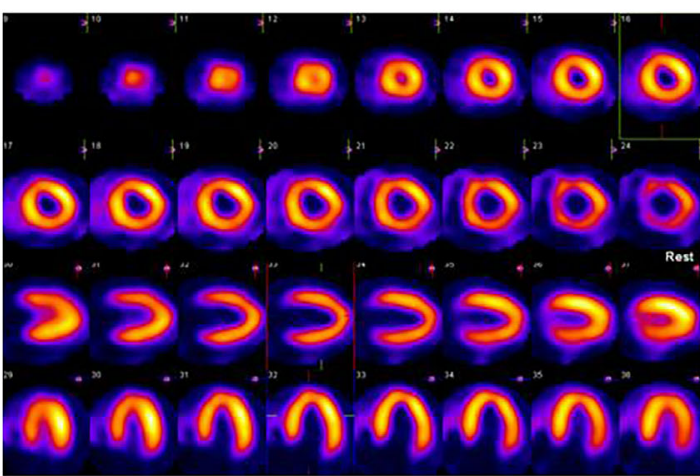

Rest image

Figure 3. Adenosine stress-rest ${ }^{99 \mathrm{~m}}$ Tc-tetrofosmin SPECT study revealed anterior myocardial silent ischemia and myocardial damage before the administration of nicorandil.

\section{6 months later after nicorandil administration}

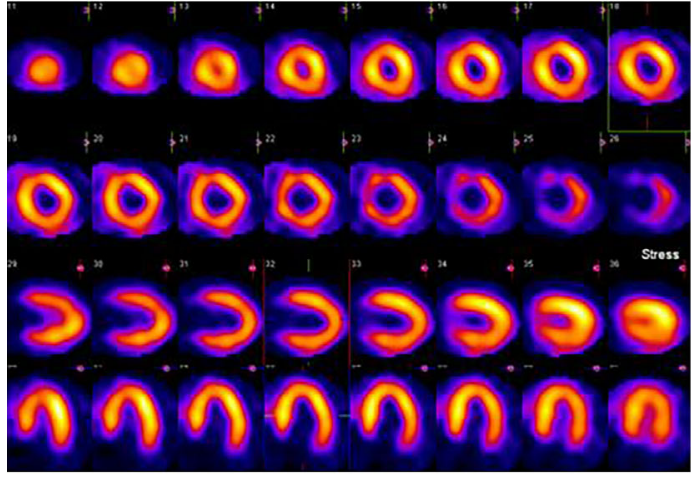

Stress image

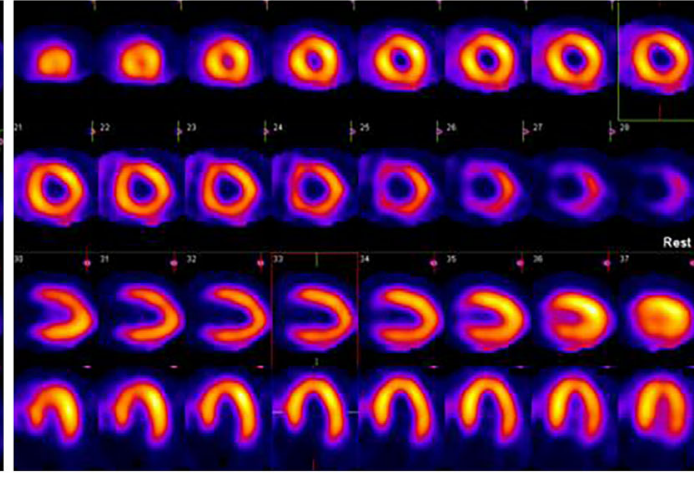

Rest image

Figure 4. The adenosine stress-rest ${ }^{99 \mathrm{~m}}$ Tc-tetrofosmin SPECT study revealed mild anterior myocardial damage without stress induced ischemia after 6 months of treatment with nicorandil.

nary vessels, such as those of $<100 \mu \mathrm{m}$ in size, via the KATP channel (4).

However, we cannot expect the large and small coronary dilatation effect of nicorandil to have an anti-ischemic effect when adenosine dilates all resistant vessels. Conversely, nicorandil can redistribute blood by dilating the coronary arteries and eventually increase the blood flow in the ischemic myocardium (5). We hypothesize that the redistribution effect on the ischemic myocardium induced by nicorandil plays a key role in inhibiting myocardial ischemia during pharmacologic stress testing with adenosine.

At the time, we believe that the probability of an increase in the visible and invisible collateral circulation flow in the jeopardized areas in response to nicorandil treatment can protect the myocardium from ischemic damage (6).

Second, the chronic vasoactive effect of the long-term administration of nicorandil on the vascular endothelial function should be discussed. Sekiya et al. documented the beneficial effects of the long-term administration of nicorandil on flow-mediated dilatation, and reported an improvement in the vascular endothelial function, which may play a role in preventing cardiovascular events (7). We hypothesize that the long-term administration of nicorandil may have resulted in the improvement of the vascular endothelial function and arteriosclerosis after the long-term administration of nicorandil, especially in the microcirculation irrigating the area of the ischemic myocardium, which might have affected the ischemic tolerance of the anterior area.

Third, we would like to discuss the direct effects of nicorandil on the intercellular mitochondria via the K-ATP channel. Some experimental studies have shown that nicorandil exerted various types of cardioprotective effects through the opening of the K-ATP channels by reducing oxidative damage, preserving the production of ATP, preventing the release of cytochrome c, modulating the properties of neutrophils, enhancing the production of prostacyclin, accelerating $\mathrm{Na}^{+}$ recovery, and attenuating the mitochondrial $\mathrm{Ca}^{2+}$ overload, with the accompanying deporalization of the mitochondrial membrane (8). Moreover, Matsuo et al. documented a cellular protective effect against ischemia due to nicorandil, inde- 
pendent of myocardial blood flow by assessing the $\Sigma$ ST during percutaneous transluminal coronary angioplasty (9). We hypothesize that the continuous administration of nicorandil can improve the ischemic tolerance of jeopardized areas through these various types of cardioprotection, even in patients with BWG syndrome.

In this case, we selected pharmacologic stress myocardial scintigraphy because we judged that the patient's exercise ability, which mainly depended on the weakness of the lower limb muscle, was not sufficient to increase the double product. However, we also believe that exercise stress myocardial scintigraphy, which can increase oxygen consumption in the myocardium, is the best strategy for detecting myocardial ischemia in patients with BWG syndrome. Thus, the anti-ischemic effect of nicorandil administration in patients with BWG syndrome may be more conspicuous in exercise stress than it is in pharmacologic stress.

In the present case, we diagnosed a typical type of BWG syndrome, as the left anterior descending artery and the left circumflex artery were visualized with a right coronary artery injection. Left coronary artery bifurcation from the pulmonary artery is a characteristic of BWG syndrome, and it has been reported that ischemia is likely to occur on the side of the anterior wall $(10,11)$. On the other hand, Katsuragi et al. reported that some BWG patients showed an inferoposterior perfusion defect - a prolonged inadequate blood supply to the right coronary region - which may cause inferoposterior cardiomyopathy (12). In the present case, the induced ischemia in the anterior area was shown by myocardial perfusion using adenosine stress and rest imaging with ${ }^{99 \mathrm{~m}}$ Tc-tetrofosmin SPECT. The location of the induced ischemia followed the typical pattern that is observed in BWG patients $(10,11)$. Moreover, the small ischemic area and a small amount of ischemic myocardium in the anterior area might allow nicorandil to more easily function as an antiischemic agent.

On the other hand, there have been reports of two potential substrates for arrhythmia in BWG patients. First, the areas with scar tissue from previous myocardial infarctions may alter the condition of the left ventricle, causing it to become arrhythmogenic. Second, arrhythmia can be triggered by an acute ischemic event during exercise, as the coronary steal phenomenon may cause inadequate perfusion (13). The fact that the area of induced ischemia was very small might have affected the patient's favorable clinical course that was observed until her admission, as no cardiac events occurred. Although we could not document the presence of subtle myocardial scar tissue without the use of cardiac magnetic resonance imaging, we did not detect the features of any large areas of arrhythmogenic cardiac tissue by cardiac echocardiography, on which they would be reflected by signs such as an increase in brightness, or thinning of the ventricular wall. However, it is possible that the induced ischemia in the anterior area could have become arrhythmogenic at a later stage. Thus, we would did not expect nicorandil to improve heart failure due to BWG syndrome be- cause the effects of nicorandil were not sufficient to improve the wall motion abnormality; rather, we suggest that its benefit was in controlling the risk of future cardiac events (such as critical arrhythmia) due to residual ischemia.

In conclusion, this case suggested that nicorandil acted as an anti-ischemic agent in an adult patient with BWG syndrome and induced ischemia, which was objectively evaluated using functional tests such as myocardial scintigraphy. We believe that nicorandil can be used in adult BWG patients [who are not undergoing coronary aortic bypass graft (CABG) or other cardiac surgery] who show objective myocardial ischemia and present contraindications to the administration of beta blockers, such as bradycardia or severe pulmonary disease.

\section{The authors state that they have no Conflict of Interest (COI).}

\section{References}

1. The Clinical Recognition of Congenital Heart Disease. 4th ed. Perloff JK, Ed. Anomalous origin of the left coronary artery rom the pulmonary trunk. WB Saunders, Philadelphia, 1994: 546-561.

2. Wesselhoeft H, Fawcett JS, Johnson AL. Anomalous origin of the heart coronary artery from the pulmonary trunk. Its clinical spectrum, pathology, and pathophysiology, based on a review of 140 cases with seven further cases. Circulation 38: 403-425, 1968.

3. Baue E, Stanley B, William S, Blakemore F. Zinsser. A later stage of anomalous coronary circulation with origin of the left coronary artery from the pulmonary artery: coronary artery steal. Circulation 36: 878-885, 1967.

4. Nakae I, Quan L, Hashimoto K, et al. Mechanism of the vasodilatory action of nicorandil on coronary circulation in dogs. Cardiovasc Drugs Ther 8: 137-145, 1994.

5. Miyazaki T, Moritani K, Miyoshi S, Asanagi M, Zhao LS, Ogawa S. Nicorandil auguments regional ischemia-induced monophasic action potential shortening and potassium acculmulation without serious proarrhythmia. J Cardiovasc Pharmacol 26: 949-956, 1995.

6. Lamping KA, Warltier DC, Hardman HF, Gross GJ. Effects of nicorandil, a new antianginal agent, and nifedipine on collateral blood flow in a chronic coronary occlusion model. J Pharmacol Exp Ther 229: 359-363, 1984.

7. Sekiya M, Sato M, Funada J, Ohtani T, Akutsu H, Watanabe K. Effects of the long-term administration of nicorandil on vascular endothelial function and the progression of arteriosclerosis. J Cardiovasc Pharmacol 46: 63-67, 2005.

8. Zhao F, Chaugai S, Chen P, Wang Y, Wang DW. Effect of nicorandil in patients with heart failure: a systematic review and metaanalysis. Cardiovasc Ther 32: 283-296, 2014.

9. Matsuo H, Watanabe S, Segawa T, et al. Evidence of pharmacologic preconditioning during PTCA by intravenous pretreatment with ATP-sensitive $\mathrm{K}^{+}$channel opener nicorandil. Eur Heart J 24: 1296-1303, 2003.

10. Finley JP, Howman-Giles R, Gilday DL, Olley PM, Rowe RF. Thallim-201myocardial imaging in anomalous left coronary artery arising from the pulmonary artery. Application before and after medical and surgical treatment. Am J Cardiol 42: 675-680, 1978.

11. Rabinovitch M, Rowland TW, Castaneda AR, Treves S. Thallium201 scintigraphy in patients with anomalous origin of the left coronary artery from the main pulmonary artery. J Pediatrics 94: 244-247, 1979.

12. Katsuragi M, Yamamoto $K$, Tashiro $T$, Nishihara $H$, Toudou $K$. Thallium-201 myocardial SPECT in Bland-White-Garland syndrome: two adult patients with inferoposterior perfusion defect. $\mathrm{J}$ 
Nucl Med 34: 2182-2184, 1993.

13. Hashimoto K, Kinoshita M, Ohbayashi Y. Coronary effects of nicorandil in comparison with nitroglycerin in chronic conscious dogs. Cardiovasc Drugs Ther 5: 131-138, 1991.
The Internal Medicine is an Open Access article distributed under the Creative Commons Attribution-NonCommercial-NoDerivatives 4.0 International License. To view the details of this license, please visit (https://creativecommons.org/licenses/ by-nc-nd/4.0/).

(C) 2017 The Japanese Society of Internal Medicine Intern Med 56: 2295-2299, 2017 